\title{
Experiência de um enfermeiro na atenção básica no interior do Amazonas: principais entraves
}

\author{
Nurse's experience in a basic care inside the Amazonas: main entrances
}

Experiencia de un enfermero en cuidado básico dentro de la amazonía: principales entradas

Lucas da Silva de Almeida ${ }^{1 *}$, Taianne Carneiro Reis ${ }^{1}$, Led Daianna Fernandes de Figueiredo", Rhuana Maria de Oliveira Pereira ${ }^{2}$, Vanessa de Souza Correia de Araújo², Adriana Duarte de Sousa ${ }^{2}$

\section{RESUMO}

Objetivo: Relatar a experiência de um Enfermeiro na Atenção Básica no interior do Amazonas. Relato de experiência: A consulta de enfermagem é feita de forma mista, entre os atendimentos dos programas e demanda livre, sobretudo por existirem várias comunidades rurais circunvizinhas que buscam atendimento quando comparecem a sede do município para receber seus benefícios sociais ou outros. Tal ação gera alta demanda de atendimento para o Enfermeiro, uma vez que não desempenha apenas essa atribuição. Durante a realização do estudo, observou-se forte pressão de demanda para o Enfermeiro, causando uma restrição de assistência domiciliar, fato esse que expressava uma atitude ambígua, levando a uma divisão de opiniões sobre a pertinência ou não da visita domiciliar como instrumento prioritário. Considerações finais: Muitas são as dificuldades enfrentadas pelo Enfermeiro, alta demanda, equipe defasada, recursos insuficientes, falta de insumos e outros. Ressaltamos que estudos como esse demonstram a necessidade de buscar melhorias, reivindicando direito de exercer aquilo que lhe compete, resultando em seu processo de trabalho otimizado.

Palavras-chave: Atenção Básica, Enfermeiro, Amazonas, Entraves.

\begin{abstract}
Objective: To report the experience of a nursing professional in primary care in the interior of Amazonas. Results: The nursing consultation is done in a mixed way, between the attendance of the programs and free demand, especially since there are several surrounding rural communities that seek care when they attend the municipality's cede to receive their social or other benefits. Such action generates a high demand for nursing care, since it does not only perform this assignment. During the study, there was strong demand pressure for the nurse, causing a restriction of home care, which expressed an ambiguous attitude, leading to a division of opinions about the pertinence or not of the home visit as a priority instrument. Conclusion: Many are the difficulties faced by the nurse, high demand, outdated staff, insufficient resources, lack of inputs and others. We emphasize that, studies like this demonstrate the need to seek improvement, claiming the right to exercise what is theirs, resulting in their optimized work process.
\end{abstract}

Key words: Basic Attention, Nurse, Amazonas, Inside.

\section{RESUMEN}

Objetivo: Reportar la experiencia de un enfermero en atención primaria en el interior de Amazonas. Informe de experiencia: La consulta de enfermería se realiza de manera mixta, entre la asistencia a los programas y

1 Universidade Federal do Amazonas (UFAM), Coari - AM. *E-mail: lucas.enf.almeida@hotmail.com

2 Universidade do Norte (UNINORTE), Manaus - AM.

SUBMETIDO EM: 8/2019

ACEITO EM: 9/2019

PUBLICADO EM: 11/2019

REAS/EJCH | Vol.11(17) | e1419 | DOI: https://doi.org/10.25248/reas.e1419.2019 Página 1 de 6 
la libre demanda, especialmente porque hay varias comunidades rurales circundantes que buscan atención cuando asisten a la cede del municipio para recibir sus beneficios sociales o de otro tipo. Dicha acción genera una alta demanda de atención de enfermería, ya que no solo se realiza esta tarea. Durante el estudio, hubo una fuerte presión de demanda para el enfermero lo que provocó una restricción de la atención domiciliaria, que expresó una actitud ambigua, lo que llevó a una división de opiniones sobre la pertinencia o no de la visita domiciliaria como instrumento prioritario. Consideraciones finales: Muchas son las dificultades que El enfermero enfrenta, la alta demanda, el personal desactualizado, los recursos insuficientes, la falta de insumos y otros. Hacemos hincapié en que, estudios como este demuestran la necesidad de buscar mejoras, reclamando el derecho a ejercer lo que es suyo, lo que resulta en su proceso de trabajo optimizado.

Palabras clave: Atención primaria, Enfermero, Amazonas, Obstaculos.

\section{INTRODUÇÃO}

O Sistema Único de Saúde (SUS) oferta o serviço de Atenção Básica (AB) a população, que é caracterizada pelas ações de promoção da saúde e prevenção de doenças, além de se constituir em primeiro nível de atendimento curativo, bem como possui amplitude para resolver problemas de saúde da maior parte da população, sendo assim, é amplamente difundida como a principal porta de entrada do usuário ao SUS (HERMIDA PMV, et al., 2015).

$A$ AB é desenvolvida com o mais alto grau de descentralização e capilaridade, executada no local mais próximo das pessoas, em meio à comunidade, sendo ela o contato preferencial dos usuários (MORENO CA, et al., 2015).

Para se chegar a esse atual modelo de $A B$, muitas mudanças ocorreram, desde a Reforma Sanitária até a criação das leis de regulamentação da saúde; por outro lado, ainda existem muitos entraves e necessita ajustar alguns pontos. Umas das principais batalhas a serem travadas é a mudança da lógica assistencial centrada na atenção secundária para ações preventivas voltadas a atenção primária a saúde (APS) (MENICUCCI TMG, 2014).

Quando se trata de profissionais que atuam na $A B$, é inerente a pessoa do Enfermeiro; desenvolve diversas ações e atividades que vão desde as assistenciais e preventivas, até as gerenciais, sendo ele um dos atores principais desse cenário de saúde (MENICUCCI TMG, 2014).

Na Unidade Básica de Saúde (UBS), o Enfermeiro desempenha seu papel em diversas áreas que adotam caráter educativo, gerencial e ainda assistência de diversos grupos, como a saúde da criança, saúde mulher, hipertensos e diabéticos, e outras gamas de áreas. É certo que essas atividades caracterizam a identidade do Enfermeiro e está voltada para onde ele desempenha esses papeis (GUEDES JS, et al., 2011; BELLAGUARDA MLR, et al., 2014).

Entretanto, o Enfermeiro encontra dificuldades no atendimento aos seus serviços, muitas vezes pela concepção conflituosa de si mesmo e da sua prática, além de outros fatores que também interferem nessa compreensão, como a não delimitação de seu espaço, a indecisão, o acúmulo de suas atribuições que prejudica as características de suas competências, bem como as diversas problemáticas regionais, como as acesso ao usuário, entre outros (FERNANDES MC, et al., 2018).

No estado do Amazonas, dadas as suas particularidades, como a grande extensão territorial, ausência de estradas, onde a principal forma de transporte é fluvial, parte da população acaba por ficar isolada em certas localidades, lançando mão de recursos mínimos para sobrevida e possuindo acesso a saúde de forma esporádica. Uma das estratégias encontradas pela população amazonense, sobretudo os mais isolados, é a utilização de recursos caseiros, como medicamentos naturais com custo reduzido e acesso fácil (CORDEIRO PM, et al., 2019).

Em busca de respostas para essas problemáticas que se apresentam, faz-se necessárias discussões acerca das atividades desenvolvidas na $A B$ pelo Enfermeiro no Amazonas e quais as reais competências 
desse profissional. Para tanto, o objetivo desse estudo é relatar a experiência de um Enfermeiro na Atenção Básica do município de Barreirinha, no interior do estado do Amazonas, Brasil, bem como expor os principais entraves da atuação que impedem assistência ideal.

\section{RELATO DE EXPERIÊNCIA}

Trata-se de um relato de experiência do tipo descritivo qualitativo, obtido durante a atuação de um Enfermeiro na Atenção Básica do município de Barreirinha no interior do estado do Amazonas no período de setembro de 2018 a março de 2019,

Na sede do município existem apenas 2 (duas) UBSs para prestar assistência a cerca de 27.355 habitantes (IBGE, 2017). A origem da população local é arraigada pelos povos quilombola e indígena.

A UBS em questão tem em sua direção uma Enfermeira. Funcionam na unidade 2 (duas) Estratégias de Saúde da Família (ESF), tendo um Enfermeiro em cada equipe e, um desses é responsável pelo Programa Nacional de Imunização (PNI) e, o outro pelos Programas de Tuberculose, Hanseníase e Leishmaniose. Além disso, funcionam os serviços médicos, odontológicos, fisioterapêuticos e do Núcleo Ampliado de Saúde da Família e Atenção Básica (NASF-AB).

Os programas disponíveis na UBS são: assistência à saúde da mulher, no planejamento familiar, pré-natal, rastreamento do câncer do colo do útero e mama, puerpério, assistência à saúde da criança, saúde do idoso, tratamento e acompanhamento de hanseníase, leishmaniose e tuberculose e imunização. As consultas de enfermagem são referentes aos programas e a demanda livre, além da realização das visitas domiciliares.

Durante a atuação como Enfermeiro na $A B$ no interior do Amazonas, as atividades desenvolvidas oportunizaram conhecer o sistema de saúde local e suas particularidades. No primeiro momento ficou nítido que se exigiria assistência diversificada e adaptável. dada a presença de povos de origem diferentes, que em sua maioria fala apenas sua língua vernácula, como é o caso dos povos indígenas. Na ausência de profissionais que dominem aquela língua, o Enfermeiro, como responsável pela equipe, teria que se desdobrar para extrair informações e estabelecer um diálogo.

A consulta de enfermagem é feita de forma mista, entre os atendimentos dos programas e demanda livre, sobretudo por existirem várias comunidades rurais circunvizinhas que buscam atendimento quando comparecem a sede do município para receber seus benefícios sociais ou outros. Tal ação gera alta demanda de atendimento para o Enfermeiro, uma vez que não desempenha apenas essa atribuição.

Ainda no que se refere à consulta de enfermagem, diferente de grandes centros de saúde, em diversas regiões no interior do Amazonas ainda é utilizado o prontuário impresso para realização de evoluções e registros, exigindo grande dispêndio de tempo do profissional.

Durante a realização do estudo, observou-se forte pressão de demanda para o Enfermeiro, causando uma restrição de assistência domiciliar, fato esse que expressava uma atitude ambígua, levando a uma divisão de opiniões sobre a pertinência ou não da visita domiciliar como instrumento prioritário, refletindo assim para que a maioria das ações fossem intramuros.

Apesar do Ministério da Saúde (MS) por meio da Política Nacional de Atenção Básica (PNAB) (2017) delimitar as atribuições do Enfermeiro, esse profissional ainda encontra dificuldades no atendimento aos seus serviços, seja pela concepção conflituosa de sua identidade ou não delimitação do seu espaço e entendimento de suas atribuições, acabando por incorporar outras atribuições de profissionais ausentes ou não, principalmente para suprimir as lacunas que rotineiramente ocorrem no dia-a-dia da unidade de saúde.

Foi observado que é rotineiro por parte do Enfermeiro assumir atribuições de outros profissionais, sobretudo atribuições médicas durante as consultas, principalmente de demanda espontânea, seja no que se refere a prescrições de medicamentos não previstas em protocolos, solicitações de exames e até mesmo condutas de tratamento. Isso acontece por diversos fatores, onde elenco o baixo número de Enfermeiros e Médicos, sobrecarga de atendimento e profissionais médicos que se recusam muitas vezes, atender um número maior de usuários que não esteja previsto. 
O artigo 79, capítulo III do Código de Ética dos Profissionais de Enfermagem (CEPE) (2017), diz que é proibido ao Enfermeiro prescrever medicamentos que não estejam estabelecidos em programas de saúde pública e/ou em rotinas aprovadas em instituições de saúde, exceto em situações de emergência. Sendo passivo de imposição de penalidades pelo Conselho Regional de Enfermagem (COREN) ou pelo Conselho Federal de Enfermagem (COFEN) a depender da infração cometida.

Uma das atribuições do Enfermeiro na $A B$ é a realização de educação em saúde, continuada e permanente (PNAB, 2017), mas na prática pouco funciona. Foram observados pelo menos 2 (dois) fatores que influenciam nesse déficit: quadro de profissionais diminuído e alta demanda de atendimento que reflete em tempo livre escasso.

Além disso, notou-se que a comunidade traz heranças, crenças e costumes locais que fazem com que se tenha aí um grande desafio para desmistificar ações que não são adequadas à saúde e podem interferir no processo terapêutico, sempre respeitando os costumes e cultura local. Um exemplo disso foi um relato, durante uma educação em saúde voltada para acidentes ofídicos - frequente nessa região - onde um dos usuários relatou que é comum ingerir combustível para se "cortar o efeito" da peçonha, fato esse que pode levar a intoxicação da vítima.

Outra problemática encontrada, que interfere diretamente na assistência, é a falta de insumos, medicamentos, instrumentos, produtos, aparelhos tecnológicos e outros. Por vezes o Enfermeiro retira do seu salário para repor algo que está em falta.

Todos esses fatores somados, excesso de atividades, sobrecarga de trabalho, limitações da autonomia, dentre outros, recaem sobre o Enfermeiro, gerando aspectos negativos da profissão e até desenvolver doenças ocupacionais, como "burnout", que para França TLB, et al (2014) é caracterizada pelo estresse excessivo que geral um esgotamento profissional, físico e mental. Tais características foram encontrados em alguns profissionais.

\section{DISCUSSÃO}

A busca pela ideal atuação do Enfermeiro na $A B$ é marcada por conflitos e, quando se trata dessa atuação no interior do estado do Amazonas, encontram-se inúmeras dificuldades. Por outro lado, o profissional desenvolve meios para levar uma assistência de qualidade aos usuários e isso faz com que ele ganhe espaço no cenário de trabalho. Para Ferreira SRS, et al (2018) quando aborda a complexidade do trabalho do enfermeiro na $A B$, destaca algumas ações que são inerentes a profissão, como a organização do serviço, supervisão das atividades exercidas por agentes de saúde e aos cuidados desenvolvidos por membros da equipe de enfermagem.

É apontado por Ferreira SRS, et al (2018) que uma das características do cotidiano do Enfermeiro é principalmente a sobrecarga de trabalho e acúmulo de diversas funções, gerando assim afastamento da assistência ao usuário, sobretudo a consulta de enfermagem. O resultado encontrado pelo autor corrobora em partes com os apresentados por esse estudo, onde, a experiência destacada mostra que os Enfermeiros da $A B$ do Amazonas, além de apresentarem um acúmulo de funções, somam a isso uma alta demanda de atendimento, não se afastando da assistência ao usuário.

Apesar de o Enfermeiro ganhar espaço no cenário de trabalho, o seu papel até então é muito confuso por conta da série de atribuições que assumi. Além da coordenação da equipe de ESF, acaba por ficar responsável por mais 2 (dois) ou 3 (três) programas da $A B$, causando uma sobrecarga de trabalho, como já vimos; acompanhado a isso, surge a tomada para si, muitas vezes, de atribuições de outros profissionais pertencentes a outras categorias, principalmente a médica, onde realiza consultas mais especificas e até mesmo prescrições que fogem daquilo que está em manuais e protocolos (FERNANDES MC, et al., 2018).

Notamos em nosso estudo, que um dos principais atores que interfere no cotidiano do Enfermeiro é o acúmulo de funções, a sobrecarga de trabalho e, dentre as problemáticas ocupacionais que isso pode causar, destacamos a Síndrome de Burnout, que França TLB, et al (2014) chama de Síndrome do Esgotamento 
Profissional e, o autor ainda aponta que é uma resposta do organismo, uma vez que está exposto a tensão crônica oriunda do contato exagerado com outras pessoas e grandes responsabilidade.

Verificou-se também que os problemas relacionados a estrutura da unidade como, falta de equipamentos e insumos são evidentes, diferentemente de grandes centros de saúde, um exemplo básico é a ausência do prontuário eletrônico obrigando o profissional a utilizar prontuário ainda impresso. Resultados parecidos foram encontrados nos estudos de Matumoto S, et al (2011), Nauderer TM e Lima MADS (2009), e Caçador BS, et al (2015), que explicitaram as dificuldades e limites da atuação do enfermeiro, enumerando a disparidade das condições estruturais em diferentes unidades de saúde como, falta de equipamento e insumos adequados.

Constatou-se que apensar da Política Nacional de Atenção Básica (2017) estabelecer que o Enfermeiro dever realizar educação em saúde, essa atividade pouco acontece, seja por escassez de profissional, sobrecarga de trabalho ou até mesmo o profissional não se sentir apto pedagogicamente. Moreno CA, et al (2015) ressalta que a educação em saúde é um desafio a ser superado e que sua importância está pautada, entro outros pontos, em fazer que a comunidade entenda como prevenir-se, porém, é superada facilmente por outra atividade sempre ficando em segundo plano, deixada para outro momento.

\section{CONSIDERAÇÕES FINAIS}

A finalidade desse estudo foi de conhecer os principais entraves que o Enfermeiro encontra atuando na $A B$ no interior do estado do Amazonas. Acredita-se que é imprescindível novos ares ou reconstruir o modelo de trabalho na $A B$ e o resgate da caracterização dela, de promoção de saúde individual ou coletiva que abranja promoção, proteção, prevenção, tratamento, reabilitação, redução de danos e manutenção à saúde de forma integral. Salienta-se que muitas são as dificuldades enfrentadas pelo Enfermeiro, a alta demanda, equipe defasada, recursos insuficientes, falta de insumos e outros. Com isso, ressalta-se que estudos como esse demonstram a necessidade de o profissional de enfermagem buscar melhorias, reivindicando direito de exercer aquilo que the compete, resultando em seu processo de trabalho otimizado e refletindo na assistência de qualidade. Ainda, faz-se necessário um estudo mais aprofundado sobre o estresse ocupacional com esses profissionais. Portando, considera-se que é oportuno uma mudança estrutural na saúde para benefício dos profissionais e da população.

\section{AGRADECIMENTOS}

Agradecemos a Secretaria de Saúde do Município e a todos os profissionais envolvidos.

\section{REFERÊNCIAS}

1. BELLAGUARDA MLR, et al. Identidade da profissional enfermeira caracterizada numa revisão integrativa. Enferm Foco. 2014;2(3):180-3.

2. BRASIL. Ministério da Saúde. Gabinete do Ministro. Portaria no 2.436, de 21 de setembro de 2017. Aprova a Política Nacional de Atenção Básica (PNAB), estabelecendo a revisão de diretrizes para a organização da Atenção Básica, no âmbito do Sistema Único de Saúde (SUS). Diário Oficial da União: Brasília, DF, 22 set. 2017.

3. CAÇADOR BS, et al. Being a nurse in the Family health strategy programme: challenges and possibilities. Rev Min Enferm [Internet]. 2015[cited 2017 Jun 01];19(3):612-26.

4. CONSELHO FEDERAL DE ENFERMAGEM. Resolução ํㅜ564/2017. Aprova o novo Código de Ética dos Profissionais de Enfermagem. Diário Oficial da União: Brasília, DF, 2017.

5. CORDEIRO PM, et al. Efeito da Justicia acuminatissima, Sara Tudo do Amazonas na Injúria Renal Aguda Isquêmica: estudo experimental. Rev Esc Enferm USP. 2019;53:e03487.

6. FERNANDES MC, et al. Identidade do enfermeiro na Atenção Básica: percepção do "faz tudo". Rev Bras Enferm. 2018;71(1):154-9.

7. FERREIRA SRS, et al. A complexidade do trabalho do enfermeiro na Atenção Primária a Saúde. Rev Bras Enferm [Internet]. 2018;71(supl1):752-7.

8. FRANÇA TLB, et al. Síndrome de Burnout: características, diagnostico, fatores de risco e prevenção. Ver. Enfem. UFPE on line., Recife, 8(10): 3539-46, out., 2014. 
9. GUEDES JS, et al. A implantação do Programa de Saúde da Família (PSF) no estado de São Paulo (1995-2002). Saúde Soc. $2011 ; 20(4): 875-883$.

10. HERMIDA PMV, et al. Metodologia ativa de ensino na formação do enfermeiro: inovação na Atenção Básica. Rev Enferm UFSM 2015 Out./Dez.;5(4):683-691.

11. INSTITUTO BRASILEIRO DE GEOGRAFIA E ESTATÍSTICA - IBGE. Barreirinha, Amazonas. 2017.

12. MATUMOTO $S$, et al. Nurses' clinical practice in primary care: a process under construction. Rev Latino-Am Enfermagem [Internet]. 2011 [cited 2017 Jun 01];19(1):123-30.

13. MENICUCCI TMG. História da Reforma Sanitária brasileira e do Sistema Único de Saúde: mudanças, continuidade e a agenda atual. História, Ciência, Saúde - Manguinhos. 2014;(21) 1:77-92.

14. MORENO CA, et al. Atribuições dos profissionais de enfermagem na Estratégia de Saúde da Família, uma revisão das Normas e Práticas. R bras ci Saúde 19(3):233-24-, 2015.

15. NAUDERER TM, LIMA MADS. Nurses' practices at health basic units in a city the South of Brazil. Rev Latino-Am Enfermagem [Internet]. 2008 [cited 2017 Jun 01];16(5):889-94.

16. POGHOSYAN L, et al. Revisiting Scope of Practice Facilitators and Barriers for Primary Care Nurs Pratitionrs: a qualitative invstigation. Policy Polit Nurs Pract. 2013;14(1):6-15.

17. SANTOS DAS, VANDENBERGHE L. Atuação profissional e bem-estar em enfermeiros. Rev Enferm UFSM. 2013;3(1):26-34.

18. SILVA LMS, et al. Convory model to Family by nurses in primary health care: thr descriptive study. O Braz $\mathrm{J}$ Nurs. $2016 ; 15(1): 52-60$. 